\title{
Nutraceutical Treatment of Patients with Polycystic Ovary Syndrome
}

\author{
Comhaire Frank ${ }^{1 *}$, Decleer Wim² \\ ${ }^{1}$ Ghent University Hospital, Consultant Endocrinologist, Fertility-Belgium Clinic, Aalter, Belgium \\ ${ }^{2}$ Head of Department of Reproductive Medicine AZ Jan Palfijn, Ghent, Belgium \\ Email: ^frank@comhaire.com, ^frank.comhaire@gmail.com
}

How to cite this paper: Frank, C. and Wim, D. (2021) Nutraceutical Treatment of Patients with Polycystic Ovary Syndrome. Open Journal of Obstetrics and Gynecology, 11, 1117-1124.

https://doi.org/10.4236/ojog.2021.119105

Received: August 9, 2021

Accepted: September 5, 2021

Published: September 8, 2021

Copyright (c) 2021 by author(s) and Scientific Research Publishing Inc. This work is licensed under the Creative Commons Attribution International License (CC BY 4.0).

http://creativecommons.org/licenses/by/4.0/

(c) (i) Open Access

\begin{abstract}
Polycystic Ovary Syndrome (PCOS) is a common cause of infertility, requiring antioestrogen and, possibly, gonadotropin treatment for the induction of ovulation. However, patients suffering from this disease need long-term treatment since PCOS is associated with an increased risk of metabolic syndrome, hyperinsulinemia, oxidative stress, inflammation, cardiovascular disease, and cancer. Allopathic treatment includes the continuous use of oral contraceptives, preferentially with complementary anti-androgen activity, and metformin. However, these treatments only partially correct all aspects of the disease, while carrying potential adverse effects per se. Complementary and alternative medicine (CAM) offers opportunities for treatment through the use of nutraceuticals containing plant extracts, minerals, and vitamins with antioxidant, anti-inflammatory, and adaptogen capacity, as well as for correcting insulin resistance. Here we justify the formulation of the nutraceutical QALY ${ }^{\circledast}$ (Jona Pharma, Elversele, Belgium) that is composed of Ubiquinone Q10, the vitamins B9 and B12, the salts of zinc and selenium, the biomass of Haematococcus pluvialis, and the extracts of Mediterranean pine bark and of Rhodiola rosea. This can be complemented by phytotherapy with the extract of Momordica charantia plus alfa lipoic acid (Momordica plus ${ }^{\circledast}$ ).
\end{abstract}

\section{Keywords}

Polycystic Ovary Syndrome

\section{Introduction}

PCOS stands for polycystic ovary syndrome describing a disease that is common in young women. Aside from the fact that this disease causes impaired fertility, there are major health hazards associated with it, including life-long hormone 
disruption, insulin resistance and metabolic syndrome, oxidative stress, chronic inflammation, and epigenetic alterations. These may result in dermatologic problems associated with hyperandrogenism, obesity, cardiovascular disease, diabetes and-probably - an increased risk of cancer.

Whereas allopathic medication, such as antioestrogens and aromatase inhibitors, sometimes combined with gonadotropins, are needed to restore fertility, hyperinsulinemia is commonly treated with metformin. However, these treatments do not address the many other health threatening aspects of PCOS, and their long-term use may cause undesired side effects.

The alternative approach, using food supplements, has been studied extensively. The scientific evidence that has emanated from these studies has been implemented into the development of the food supplement QALY ${ }^{\oplus}$ (Jona Pharma, Elversele, Belgium) that consists of a judicious mixture of plant extracts, minerals, vitamins, and fatty acids, acting in synergism. The rationale for this formulation is explained and summarized in the present paper.

\section{Materials and Methods}

\subsection{Clinical Trials}

Over the last two decades, a large number of patients have participated in controlled or pragmatic trials using either the $\mathrm{QALY}^{\circledR}$ supplement, or its separate constituents, for the treatment of male [1] or female infertility [2]. Overall the complementary intake of $\mathrm{QALY}^{\circledast}$ increased the spontaneous pregnancy rate and the success rate of intra-uterine insemination (IUI) of infertile couples with number needed to treat of 4 . When given before conception of In Vito Fertilization (IVF) the clinical pregnancy rate increased from $35 \%$ up to $50 \%$ per treatment cycle [3]. Two out of every 3 patients suffering from myalgic encephalomyelitis/chronic fatigue syndrome (ME/CFS) or from the post-COVID syndrome (also called "long-COVID") [4] experienced benefit from this nutraceutical with the result of the Fatigue Severity Scale significantly decreasing by an average of $14 \%$ after one month. QALY $^{\oplus}$ was also given with the aim to correct the presumed mechanisms of ageing [5] and to prevent, or delay the progression of Alzheimer dementia [6].

Momordica plus ${ }^{\oplus}$ prescribed to patients with the metabolic syndrome or type II diabetes, reduced insulin resistance during follow-up of up to 6 years in over $90 \%$ of cases [1].

\subsection{Literature Study}

The results of these personal pragmatic studies were challenged against scientific publications recruited through search in the usual electronic programs (Pubmed, Google) and associated references. The considerations based on our clinical experience, as described in the references cited above, and on the literature search are given in separate paragraphs related to the intended biological effects being relevant to the pathology of PCOS. 


\section{Considerations}

\subsection{Oxidative Stress and Anti-Oxidants}

Oxidative stress is an important contributing factor to the pathogenesis of PCOS [7] [8]. Antioxidant treatment has, therefore, been recommended in these patients [9].

The biomass of the microscopic algae Haematococcus pluvialis is a natural source of Astaxanthin, which is a strong antioxidant, with complementary anti-inflammatory activity. Astaxanthin belongs to the group of the carotenoids, and it has no toxic nor teratogenic effects. It protects against oxidative damage to the cell membrane and to DNA, and has favourable effects in various diseases [10].

The oxido-reductase co-enzyme ubiquinol Q10 (CoQ-10) mainly acts as an antioxidant at the mitochondrial level and improves their function [11]. CoQ-10 has successfully been given for the treatment of patients with PCOS [12].

\subsection{Metabolic Syndrome and Inflammation}

The extract of the bark of the Mediterranean pine tree (pine bark extract) is rich in flavonoids, anthocyanidins in particular. Pine bark extract exerts strong activity against inflammation [13], which is an important aspect of PCOS [14], and has anti-oxidant effects as well.

Many patients with PCOS suffer from insulin resistance. Pine bark extract improves this condition [15] and reduces the health risks associated with it [16].

In PCOS patients with the metabolic syndrome, either or not associated with obesity, Momordica charantia extract improves cellular insulin receptivity by activating the adenosine monophosphate-activate protein kinase (AMPK). Its efficacy is enhanced by alfa lipoic acid that facilitates multiple steps of glucose/pyruvate metabolism at the post-receptor level [17].

\subsection{Hormone Deregulation and Adaptogens}

Hyperandrogenism, imbalance of the gonadotropin secretion, and deregulation of the hypothalamic-pituitary-adrenal (HPA) axis are well-known and typical endocrine phenomena in the majority of PCOS patients [18]. The extract of Rhodiola rosea is a strong adaptogen attenuating adrenal hypersecretion through several pathways [19].

\subsection{Homocysteine and Vitamins B9 \& 12}

The plasma homocysteine concentration is commonly increased in PCOS patients [20]. Administration of B-group vitamins [21], folic acid in particular [22], reduces circulating homocysteine in patients with PCOS.

Elevated serum homocysteine is associated with increased homocysteine concentration in follicular fluid, which affects oocyte fertilization [3]. In addition, hyper-homocystëinemie is an independent risk factor for cardiovascular disease and osteoporosis, which are both more common in PCOS patients [23]. 


\subsection{Minerals: Selenium and Zinc}

Selenium-dependant glutathione peroxidase has anti-tumour properties [24] and is relevant to PCOS patients [25]. Indeed, lower levels of selenium have been found in women with PCOS [26]. Food supplementation with selenium-salt was associated with improved metabolic response in these patients [27], and might reduce cancer risk.

Mitochondrial dysfunction and long-term metabolic imbalance are present in many PCOS patients [11] [28]. This can be corrected by zinc supplementation [29], especially when given together with Ubiquinol Q10.

\subsection{Omega-3 Fatty Acids}

The long-chain poly-unsaturated (PUFA) omega-3 fatty acids docosohexaenoic acid (DHA) and eicosapentaenoic acid (EPA) do attenuate PCOS complications through a number of mechanisms [30]. Krill oil is a rich source of these PUFA's that are esterified in phospholipids. The oral absorption of esterified DHA and EPA is better than that of the pure fatty acids, and Krill oil is more environmental friendly than fish oil.

\subsection{Methylation and Epigenetics}

Recently the importance of epigenetic interactions between microRNA and DNA methylation has been highlighted in patients PCOS [31]. Hypermethylation of certain DNA domains was found to play a pivotal role, as it does in many diseases and in carcinogenesis [32].

Food supplementation with QALY $^{\oplus}$ reduces the methylation of the human telomerase reverse transcriptase (hTERT)-promoter region by an average of $14 \%$. In particular, the second dinucleotide (CpG2), which has been associated with an increased risk of several types of cancers [3], is lowered. It can be speculated that decreased methylation, in synergism with reduced hyperinsulinemia and increased selenium-dependant glutathione peroxidase activity will benefit PCOS patients and will lower their risk of tumorigenesis.

\section{Discussion}

Patients suffering from PCOS are at risk of long-term negative health hazards that are not, or only partly addressed by the usual allopathic treatments, the adherence to which is suboptimal in real-world [33]. In addition, Metformin may induce Vitamin B12 deficiency [34]. The mechanisms underlying PCOS-associated health hazards must be targeted and corrected, in order to improve the wellbeing of patients and to reduce the probability of ill-health when aging.

Nutraceutical food supplementation using natural ingredients is devoid of adverse effects, and the judiciously formulated composition presented here uses synergism of several constituents to counteract unfavourable biological processes.

The experience gained from previous clinical trials, together with ample data 
from the scientific literature, sustain the positive benefit/risk ratio of the QALY ${ }^{\odot}$ nutraceutical and of the phytotherapy with Momordica plus'. These products should be considered as add-on for patients with PCOS and implemented from menarche to the menopause.

The biological effectivity can be monitored through periodic measurement of e.g. malondialdehyde in urine as a biomarker of oxidative stress and of the risk of cancer as well as of cardiovascular disease [35]. Glycosylated haemoglobin A1c and C-peptide concentration in blood [36] can serve as measures of glucose metabolism and insulin resistance.

\section{Conclusion}

Critical review of the effects of nutritional supplementation of POCS patients [37] with the nutraceutical QALY $^{\circledast}$ and phytotherapy with Momordica plus ${ }^{\oplus}$ sustain the potential benefit of the synergism of their components on the pathogenic mechanisms causing health hazards to patients with this disease. The judicious formulation using natural components should protect these patients from ill-health that is commonly associated with the polycystic ovary syndrome, while being devoid of adverse side effects.

\section{Formulations}

One package of QALY contains two separate flasks with each 60 capsules of QALY1 and QALY 2, which is sufficient for 2 months treatment. The product is commercially available and can be ordered from Jona Pharma Ltd. (Dorpstraat, 61, B9140 Elversele, Belgium) https://jonapharma.com.

\section{QALY 1}

\section{Composition per capsule:}

Rhodiola rosea $175 \mathrm{mg}$; Haematococcus pluvialis $97.5 \mathrm{mg}$; Coenzyme Q10 25 mg; Pinus maritima $21 \mathrm{mg}$; Zinc $3 \mathrm{mg}$; Folic acid $200 \mathrm{mcg}$; Selenium $70 \mathrm{mcg}$; Vitamin B12 $1.25 \mathrm{mcg}$.

Take one capsule per day after breakfast.

\section{QALY 2}

Composition per capsule (gelsolet):

Lipid extract from Antarctic Krill (Euphausia superba) $500 \mathrm{mg}$ (60 mg EPA and $5 \mathrm{mg}$ DHA).

Take one capsule per day after breakfast, together with QALY 1.

\section{Momordica Plus}

Composition per capsule:

Extract 16:1 of bitter melon (Momordica charantia) $400 \mathrm{mg}$; Alpha lipoic acid $100 \mathrm{mg}$.

Take one or two capsules per day, as prescribed by your doctor. 


\section{Conflicts of Interest}

The first author (FC) holds the Belgian patent on the fixed proportion of the combination of the 3 following ingredients present in QALY1: Selenomethionine, Astaxanthin and Mediterranean pine bark extract.

\section{References}

[1] Comhaire, F. (2015) Treating Hyperinsulinemia with Momordica Charantia. Journal of Metabolic Syndrome, 4, 3.

[2] Comhaire, F. (2010) The Role of Food Supplementation in the Treatment of the Infertile Couple and for Assisted Reproduction. Andrologia, 42, 331-340. https://doi.org/10.1111/j.1439-0272.2009.01025.x

[3] Decleer, W., Comhaire, F., De Clerck, K., et al. (2020) Preconception Nutraceutical Food Supplementation Can Prevent Oxidative and Epigenetic DNA Alterations Induced by Ovarian Stimulation for IVF and Increases Pregnancy Rates. Facts, Views \& Vision in ObGyn, 7, 23-30.

[4] Comhaire, F. and Deslypere, J.P. (2020) News and Views in Myalgic Encephalomyelitis/Chronic Fatigue Syndrome (ME/CFS): The Role of Co-Morbidity and Novel Treatments. Medical Hypotheses, 134, Article ID: 109444. https://doi.org/10.1016/j.mehy.2019.109444

[5] Comhaire, F. and Decleer, W. (2020) Can the Biological Mechanisms of Ageing Be Corrected by Food Supplementation. The Concept of Health Care over Sick Care. The Aging Male, 23, 1146-1157. https://doi.org/10.1080/13685538.2020.1713080

[6] Comhaire, F. (2020) Novel Perspectives for Treating and Preventing Dementia by Complementary Medicine. International Journal of Clinical Studies \& Medical Cases, 4, 1-6. https://doi.org/10.46998/IJCMCR.2020.04.000083

[7] Zuo, T., Zhu, M. and Xu, W. (2016) Roles of Oxidative Stress in Polycystic Ovary Syndrome and Cancers. Oxidative Medicine and Cellular Longevity, 2016, Article ID: 8589318. https://doi.org/10.1155/2016/8589318

[8] Mohammadi, M. (2019) Oxidative Stress and Polycystic Ovary Syndrome: A Brief Review. International Journal of Preventive Medicine, 10, 86. https://doi.org/10.4103/ijpvm.IJPVM_576_17

[9] Amini, L., Tehranian, N., Movahedin, M., et al. (2015) Antioxidants and Management of Polycystic Ovary Syndrome in Iran: A Systematic Review of Clinical Trials. Iranian Journal of Reproductive Medicine, 13, 1-8.

[10] Ekpe, L., Inaku, K. and Ekpe, V. (2018) Antioxidant Effects of Astaxanthin in Various Diseases-A Review. Journal of Molecular Pathophysiology, 7, 1-6. https://doi.org/10.5455/jmp.20180627120817

[11] Dabravolski, S., Nikiforov, N., Eid, A.H., et al. (2021) Mitochondrial Dysfunction and Chronic Inflammation in Polycystic Ovary Syndrome. International Journal of Molecular Sciences, 22, 3923. https://doi.org/10.3390/ijms22083923

[12] Izadi, A., Ebrahimi, S., Shirazi, S., et al. (2019) Hormonal and Metabolic Effects of Coenzyme Q10 and/or Vitamin E in Patients with Polycystic Ovary Syndrome. The Journal of Clinical Endocrinology \& Metabolism, 104, 319-327. https://doi.org/10.1210/jc.2018-01221

[13] D’Andrea, G. (2010) Pycnogenol: A Blend of Procyanidins with Multifaceted Therapeutic Applications? Fitoterapia, 81,724-736. https://doi.org/10.1016/j.fitote.2010.06.011 
[14] Shorakae, S., Ranasinha, S., Abell, S., et al. (2018) Inter-Related Effects of Insulin Resistance, Hyperandrogenism, Sympathetic Dysfunction and Chronic Inflammation in PCOS. Clinical Endocrinology, 89, 628-633. https://doi.org/10.1111/cen.13808

[15] Gulati, O.P. (2015) Pycnogenol ${ }^{\odot}$ in Metabolic Syndrome and Related Disorders. Phytotherapy Research Phytotherapy Research, 29, 949-968. https://doi.org/10.1002/ptr.5341

[16] Belcaro, G., Luzzi, C.R., Cesarone, M.R., et al. (2013) Pycnogenol ${ }^{\circledR}$ Supplementation Improves Health Risk Factors in Subjects with Metabolic Syndrome. Phytotherapy Research, 10, 1572-1578. https://doi.org/10.1002/ptr.4883

[17] Golbidi, S., Badran, M. and Laher, I. (2011) Diabetes and Alpha Lipoic Acid. Frontiers in Pharmacology, 2, 69. https://doi.org/10.3389/fphar.2011.00069

[18] Goodarzi, M.O., Carmina, E. and Azziz, R. (2015) DHEA, DHEAS and PCOS. The Journal of Steroid Biochemistry and Molecular Biology, 145, 213-225. https://doi.org/10.1016/j.jsbmb.2014.06.003

[19] Panossian, A., Hambardzumyan, M., Hovhanissyan, A., et al. (2007) The Adaptogens Rhodiola and Schizandra Modify the Response to Immobilization Stress in Rabbits by Suppressing the Increase of Phosphorylated Stress-Activated Protein Kinase, Nitric Oxide and Cortisol. Drug Target Insights, 2, 39-54. https://doi.org/10.1177/117739280700200011

[20] Loverro, G., Lorusso, F., Mei, L., at al. (2002) The Plasma Homocysteine Levels Are Increased in Polycystic Ovary Syndrome. Gynecologic and Obstetric Investigation, 53, 157-162. https://doi.org/10.1159/000058367

[21] Kilicdag, E.B., Bagis, T., Tarim, E., et al. (2005) Administration of B-Group Vitamins Reduces Circulating Homocysteine in Polycystic Ovarian Syndrome Patients Treated with Metformin: A Randomized Trial. Human Reproduction, 20, 1521-1528. https://doi.org/10.1093/humrep/deh825

[22] Kazerooni, T., Asadi, N., Dehbashi, S., et al. (2008) Effect of Folic Acid in Women with and without Insulin Resistance Who Have Hyperhomocysteinemic Polycystic Ovary Syndrome. International Journal of Gynecology \& Obstetrics, 101, 156-160. https://doi.org/10.1016/j.ijgo.2007.10.024

[23] Yang, H.Y., Lee, H.S., Huang, W.T., et al. (2018) Increased Risk of Fractures in Patients with Polycystic Ovary Syndrome: A Nationwide Population-Based Retrospective Cohort Study. Journal of Bone and Mineral Metabolism, 36, 741-748. https://doi.org/10.1007/s00774-017-0894-8

[24] Doroshow, J.H. and Juhasz, A. (2019) Modulation of Selenium-Dependent Glutathione Peroxidase Activity Enhances Doxorubicin-Induced Apoptosis, Tumour Cell Killing and Hydroxyl Radical Production in Human NCI/ADR-RES Cancer Cells Despite High-Level P-Glycoprotein Expression. Free Radical Research, 53, 882-891. https://doi.org/10.1080/10715762.2019.1641602

[25] Yin, W., Falconer, H., Yin, L., et al. (2019) Association between Polycystic Ovary Syndrome and Cancer Risk. JAMA Oncology, 5, 106-1017.

https://doi.org/10.1001/jamaoncol.2018.5188

[26] Coskun, A., Arikan, T., Kilinc, M., et al. (2013) Plasma Selenium Levels in Turkish Women with Polycystic Ovary Syndrome. European Journal of Obstetrics, Gynecology and Reproductive Biology, 168, 183-186. https://doi.org/10.1016/j.ejogrb.2013.01.021

[27] Jamilian, M., Razavi, M., Kashan, Z., et al. (2015) Metabolic Response to Selenium Supplementation in Women with Polycystic Ovary Syndrome: A Randomized, 
Double-Blind, Placebo-Controlled Trial. Clinical Endocrinology, 82, 885-891. https://doi.org/10.1111/cen.12699

[28] Zhang, J., Bao, Y., Zhou, X., et al. (2019) Polycystic Ovary Syndrome and Mitochondrial Dysfunction. Reproductive Biology and Endocrinology, 17, Article No. 67. https://doi.org/10.1186/s12958-019-0509-4

[29] Guler, I., Himmetoglu, O., Turp, A., et al. (2014) Zinc and Homocysteine Levels in Polycystic Ovary Syndrome Patients with Insulin Resistance. Biological Trace Element Research, 158, 297-304. https://doi.org/10.1007/s12011-014-9941-7

[30] Salek, M., Clark, C.C.T., Taghizadeh, M., et al. (2019) N-3 Fatty Acids as Preventive and Therapeutic Agents in Attenuating PCOS Complications. EXCLI Journal, 18, 228-575.

[31] Mao, T., Li, T., Zhao, H., et al. (2021) Identification of Epigenetic Interaction between microRNA and DNA Methylation Associates with Polycystic Ovarian Syndrome. Journal of Human Genetics, 66, 123-137. https://doi.org/10.1038/s10038-020-0819-6

[32] Baylin, S.B. and Herman, J.G. (2000) DNA Hypermethylation in Tumorigenesis: Epigenetics Joins Genetics. Trends in Genetics, 16, 168-174.

https://doi.org/10.1016/S0168-9525(99)01971-X

[33] Campbell, D.J.T., Campbell, D.B., Ogundeji, Y., et al. (2021) First-Line Pharmacotherapy for Incident Type 2 Diabetes: Prescription Patterns, Adherence and Associated Costs. Diabetic Medicine, 38, e14622. https://doi.org/10.1111/dme.14622

[34] Martin, D., Thaker, J., Shreve, M., et al. (2021) Assessment of Vitamin $B_{12}$ Deficiency and B Screening Trends for Patients on Metformin: A Retrospective Cohort Case Review. BMJNutrition, Prevention \& Health, 4, 30-35. https://doi.org/10.1136/bmjnph-2020-000193

[35] Bannigida, D.M., Nayak, B.S. and Vijayraghavan, R. (2020) Insulin Resistance and Oxidative Marker in Women with PCOS. Archives of Physiology and Biochemistry, 126, 183-186. https://doi.org/10.1080/13813455.2018.1499120

[36] Jones, A.G. and Hatterslay, A.T. (2013) The Clinical Utility of C-Peptide Measurement in the Care of Patients with Diabetes. Diabetic Medicine, 30, 103-117. https://doi.org/10.1111/dme.12159

[37] Gunalan, E., Yaba, A. and Yilmaz, B. (2018) The Effect of Nutrient Supplementation in the Management of Polycystic Ovary Syndrome-Associated Metabolic Dysfunctions: A Critical Review. Journal of the Turkish-German Gynecological Association, 19, 220-232. https://doi.org/10.4274/jtgga.2018.0077 